\title{
Evaluation of monocyte-derived dendritic cells, $T$ regulatory and Th17 cells in chronic myeloid leukemia patients treated with tyrosine kinase inhibitors
}

\author{
Iwona Hus ${ }^{1 *}$, Jacek Tabarkiewicz ${ }^{2 *}$, Magdalena Lewandowska ${ }^{2}$, Magdalena Wasiak ${ }^{2}$, \\ Paulina Wdowiak², Maria Kusz¹, Monika Legieć ${ }^{1}$, Anna Dmoszyńska ${ }^{1}$, Jacek Roliński² \\ ${ }^{1}$ Department of Hematooncology and Bone Marrow Transplantation, Medical University \\ of Lublin, Poland \\ ${ }^{2}$ Department of Clinical Immunology, Medical University of Lublin, Poland \\ *Equal contribution of both authors
}

\begin{abstract}
Immunotherapy with dendritic cells (DC) may constitute a new and advantageous option for patients with chronic myeloid leukemia (CML) who respond to therapy with tyrosine kinase inhibitors (TKI), but do not reach complete cytogenetic or molecular remission. In this study, we evaluated the immunophenotype of DC generated from monocytes (Mo-DC) of patients with CML and the influence of TKI therapy on the results of CML-DC generation. We also measured the percentages of T regulatory cells (Tregs) as well as Th17 cells in 19 untreated patients suffering from CML, and in 28 CML patients treated with TKI. We found that DC can be reliably generated from the peripheral blood $\mathrm{CD} 14^{+}$cells of untreated CML patients. But we observed a persistent expression of CD14 monocyte marker on DC from CML patients, together with lower percentages of Mo-DC with expression of CD1a $(p=0.002)$, CD80 $(p=0.0005)$, CD83 $(p=0.0004)$, and CD209 ( $p=0.02)$ compared to healthy donors. There was an adverse correlation between WBC count and the percentage of Mo-DC with co-expression of CD80 and CD86 $(\mathrm{R}=-0.63 ; \mathrm{p}=0.03)$. In patients treated with TKI, we observed higher efficacy of DC generation in seven-day cultures, compared to untreated patients. Expression of CD209 on DC was higher in patients treated with TKI (0.02). The duration of TKI therapy correlated adversely with MFI for CD1a $(\mathrm{R}=-0.49 ; \mathrm{p}=0.006)$ and positively with MFI for CD83 $(\mathrm{R}=0.63$; $\mathrm{p}=0.01)$. Percentages of $\mathrm{CD} 4{ }^{+} \mathrm{CD} 25^{\text {high }} \mathrm{FoxP} 3^{+}$cells $(\mathrm{p}=0.0002)$ and Th17 cells $(\mathrm{p}=0.02)$ were significantly higher in untreated CML patients compared to healthy controls. There was a significant correlation between the percentage of Treg cells and the percentage of peripheral blood basophiles $(\mathrm{R}=0.821 ; \mathrm{p}=0.02)$. There were no changes in Tregs or Th17 cell percentages in CML patients after six months of TKI therapy. However, the expression of intracellular IL-17 in Th17 cells correlated negatively with the time of TKI therapy in the whole group of treated patients $(\mathrm{R}=-0.516 ; \mathrm{p}=0.04)$. We noted a correlation between IL-6 serum level and peripheral blood WBC count $(\mathrm{R}=0.492 ; \mathrm{p}=0.04)$. There was also an inverse correlation between the serum level of IL-6 and the duration of TKI therapy $(R=-0.66 ; p=0.03)$. Taken together, our data shows that mature DC can be generated from CML patients treated with TKI, and that the yield of Mo-DC is higher in patients treated with TKI than in patients with active disease. This should encourage further trials with DC immunotherapy in patients with cytogenetic response after TKI therapy. We also found increased frequencies of T regulatory and Th17 cells in CML patients, which might suggest their potential role in immunity against
\end{abstract}

Correspondence address: I. Hus, Department of

Hematooncology, Medical University of Lublin,

Staszica Str. 11, 20-081 Lublin, Poland;

tel.: (+ 48 81) 53454 68; fax: (+ 48 81) 5345605 ;

e-mail: iwonach.hus@gmail.com 
this disease. Further studies are needed to determine if manipulation of these cell populations might improve the results of DC immunotherapy. (Folia Histochemica et Cytobiologica 2011; Vol. 49, No. 1, pp. 153-160)

Key words: chronic myeloid leukemia, tyrosine kinase inhibitors, dendric cells, T regulatory cells, Th17 cells

\section{Introduction}

Immunotherapeutic strategies have a potential to induce anti-tumor responses and are increasingly being explored in patients with hematological malignancies. Dendritic cells (DC) are professional antigen presenting cells, which are capable of initiating specific immune responses and have a potential clinical application [1]. For use in immunotherapy, dendritic cells are generated and pulsed with tumor-specific antigens under in vitro conditions [2,3]. Usually, peripheral blood monocytes are used as DC precursors [4]. In patients with myeloid leukemias, dendritic cells (DC) may originate from leukemic cells and thus may also express leukemia-specific antigens.

Chronic myeloid leukemia (CML) is a clonal disease, characterized by a reciprocal $t(9,22)$ chromosomal translocation resulting in a chimeric $\mathrm{BCR} / \mathrm{ABL}$ fusion gene that encodes an abnormal fusion protein (p210) with a tyrosine kinase activity, which probably plays a key role in the pathogenesis of the disease [5]. Imatinib mesylate is a specific inhibitor of of Abl protein tyrosine kinases, which has been shown to induce complete hematologic and cytogenetic responses in a significant proportion of patients [6] and has become the standard first-line treatment for patients with chronic phase CML (CP-CML). Novel tyrosine kinase inhibitors (TKI) with greater therapeutic potency, such as dasatinib, nilotinib and bosutinib have been introduced into the management of patients with CML resistant to imatinib.

Immunotherapy with DC might constitute a new and advantageous option for patients who respond to TKI therapy, but do not reach complete cytogenetic or molecular remission. Initial clinical trials with CML-derived DC (CML-DC) immunotherapy have been reported [7-9] and have shown immune and clinical responses in some patients [9]. The optimal vaccination protocol for inducing anti-tumor responses using DC has not yet been established, meaning that further studies into the biology of CML-DC are still required, especially in the context of the influence of TKI therapy on CML-DC. Recent data has shown that anti-tumor responses might be significantly suppressed by $\mathrm{T}$ helper cells with regulatory functions (Treg) [10], which suggests that the results of DC vaccinations might also be negatively affected by Treg [11]. More recent studies have revealed new populations of T helper cells, such as Th17 cells [12], whose role in tumor immunity remains unclear [13].

In the present study, we evaluated the immunophenotype of Mo-DC generated from patients with CML. Apart from the well known markers of DC maturation, we also measured the expression of other molecules essential for DC function. And we evaluated the influence of TKI therapy on the results of CML-DC generation. We also measured the percentages of Treg and the percentages of Th17 cells in untreated CML patients and in CML patients treated with TKI.

\section{Material and methods}

\section{Patients}

Between November 2007 and October 2009, 38 patients with CP-CML who were treated in the Hematooncology and Bone Marrow Transplantation Department of the Medical University of Lublin were recruited into the study. Twenty healthy volunteers were also recruited as a control group. The study was approved by the Local Ethics Committee and all individuals gave their informed consent. From 19 patients, blood samples were taken at diagnosis before the start of therapy. From 28 patients, samples were taken during TKI therapy. Eighteen of these patients were treated with imatinib at a dose of $400 \mathrm{mg}$, two patients were treated with imatinib at a dose of 600 $\mathrm{mg}$, four patients were treated with dasatinib at a dose of $100 \mathrm{mg}$, and four patients were treated with bosutinib at a dose of $500 \mathrm{mg}$. All the patients were in complete cytogenetic remission at sampling. The clinical characteristics of the patients are presented in Table 1.

\section{Methods}

Generation of autologous DC from the peripheral blood monocytes of CML patients

Peripheral blood mononuclear cells (PBMC) were isolated from $40 \mathrm{ml}$ heparinized peripheral blood samples by density gradient centrifugation (Gradisol-L, Aqua Medica, Lodz, Poland). After isolation, the cells were washed twice with PBS and incubated with anti- 
Table 1. Clinical characteristics of the patients participating in the study

\begin{tabular}{|l|c|}
\hline Number of patients & 38 \\
\hline Gender (M/F) & $26 / 12$ \\
\hline Age (years); mean (range) & $52.6(24.0-77.0)$ \\
\hline Number of untreated patients & 19 \\
\hline $\begin{array}{l}\text { Number of patients treated with imatinib } \\
\text { Duration of imatinib therapy (months); mean (range) }\end{array}$ & $26.2(6.0-65.0)$ \\
\hline $\begin{array}{l}\text { Number of patients treated with dasatinib } \\
\text { Duration of dasatinib therapy (months); mean (range) }\end{array}$ & 6 \\
\hline $\begin{array}{l}\text { Number of patients treated with bosutinib } \\
\text { Duration of bosutinib therapy (months); mean (range) }\end{array}$ & $10.0(4.0-20.0)$ \\
\hline
\end{tabular}

-CD14 microbeads (Miltenyi-Biotec, Bergisch Gladbach, Germany) and passed through MACS separation columns according to the manufacturer's protocols. The CD14 positive cell population was used to prepare monocyte derived dendritic cells (Mo-DC). The $\mathrm{CD}_{14}{ }^{+}$cells were cultured in the appropriate culture medium containing RPMI-1640 supplemented with $2 \%$ human albumin (Baxter), $100 \mathrm{IU} / \mathrm{ml}$ penicillin, $50 \mathrm{mg} / \mathrm{ml}$ streptomycin and $100 \mathrm{mg} / \mathrm{ml} \mathrm{neo-}$ mycin (Sigma-Aldrich, Germany). On days 1, 3 and 5 of the culture, 1,000 IU/ml rhGM-CSF (Leukine, Berlex, USA) and 500 IU/ml rhIL-4 (Miltenyi-Biotec, Bergisch Gladbach, Germany) were added. Tumor necrosis factor a $(\mathrm{TNFa})$ at a concentration of $50 \mathrm{ng} / \mathrm{ml}$ and the lysates from CD $33^{+}$leukemic cells were added during the last 48 hours of culture for the maturation and pulsing of the DC. On day 8 of the culture, DCs were harvested using Trypsin/EDTA (Biochrom AG, Germany) solution. Their viability was checked by Trypan Blue (Sigma-Aldrich, Germany) staining in light microscopy. Mature Mo-DC were evaluated for the expression of CD1a, CD14, CD80, CD83, CD86, CD206, CD209 and HLA-DR by flow cytometry.

Flow cytometry analysis of T regulatory cells

Treg lymphocytes were estimated by Human Treg Flow $^{\mathrm{TM}}$ Kit (FOXP3 Alexa Fluor® 488/CD4 PECy5/ /CD25 PE, Biolegend, USA) according to the manufacturer's protocol. Results are calculated as mean $\mathrm{CD}^{+} / \mathrm{CD} 25^{+} / \mathrm{FOXP}^{+}$cells percentage in $\mathrm{CD}^{+}$gate from triplicate counting for each sample.

Flow cytometry analysis of Th17 cells

Peripheral blood mononuclear cells (PBMCs) were isolated by Gradisol L density gradient centrifugation. Cells were cultured at $2 \times 10^{6}$ cells $/ \mathrm{ml}$ in RPMI 1640 medium supplemented with $10 \%$ FBS, penicillin and streptomycin. The cells were stimulated with ionomycin $(2 \mu \mathrm{g} / \mathrm{ml})$ and phorbol myristate acetate
(PMA: $2.5 \mu \mathrm{g} / \mathrm{ml}$ ) in the presence of brefeldin A $(2 \mu \mathrm{g} / \mathrm{ml})$ for 4 hours at $37^{\circ} \mathrm{C}$ in an atmosphere containing $5 \% \mathrm{CO}_{2}$. Subsequently, cells were transferred to tubes and washed once in PBS. Then the cells were incubated with the monoclonal antibodies against surface markers: FITC-conjugated anti-CD4 (Becton Dickinson, USA) and PE-Cy5-conjugated anti-CD3 (Becton Dickinson, USA) for 20 minutes at room temperature. Afterwards, cells were re-suspended in fixation and a permeabilization solution with Cytofix/ /Cytoperm according to the manufacturer's protocol. Then they were stained with PE-conjugated anti-IL-17 (eBioscience, USA) for 15 minutes at $4^{\circ} \mathrm{C}$. We analysed intracellular expression of IL-17 in CD4 ${ }^{+}$ cells among the lymphocyte-gate with the use of BDFACS Calibur flow cytometer and CellQuest software.

\section{Analysis of IL-6 serum level}

Serum concentration of IL- 6 was measured according to the manufacturer's protocol using human IL-6 ELISA kit (Diaclone, UK) and multilabel plate reader VICTOR $^{\mathrm{TM}}$ (PerkinElmer, USA).

\section{Statistical analysis}

Results are shown as mean value $\pm \mathrm{SD}$ and median followed by IQR (Interquartile Range). U Mann-Whitney, Wilcoxon's non-parametric tests and Spearman's rank correlation were used for statistical analysis.

\section{Results}

\section{Results of DC generation in untreated CML patients}

We found that DC could be reliably generated from the peripheral blood CD14 ${ }^{+}$cells of 19 untreated CML patients. Seven-day cultures of monocytes in the cytokines supplemented media resulted in the 


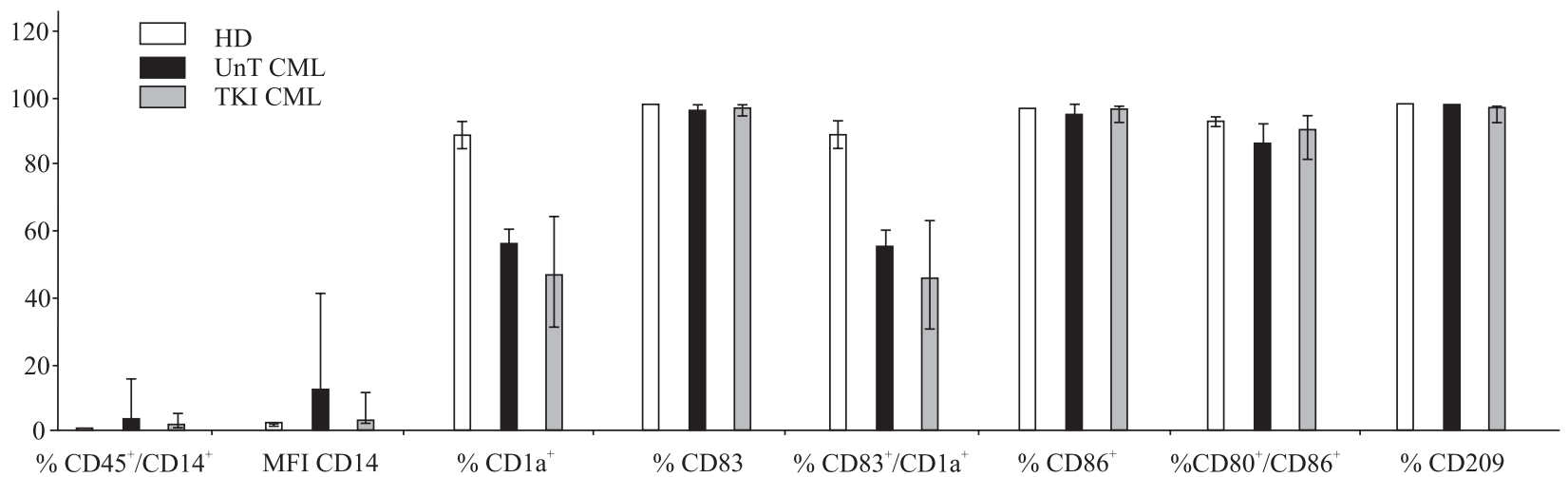

Figure 1. Values of measured parameters describing the immunophenotype of Mo-DC (UnT — untreated CML patients; TKI CML - CML patients treated with TKI; HD - healthy donors). Statistical analysis described in text

generation of cells with a veiled appearance characteristic for mature DC in light microscopy (data not shown). The mean viability of generated DC was $93 \%$ $\pm 6 \%$, and the median percentage of DC among the cells in the culture was $23 \%$ (range 3.22-82.89\%).

Analysis of the Mo-DC phenotype showed a persistent expression of CD14 monocyte marker on DC generated from monocytes of CML patients. The percentage of $\mathrm{CD} 14^{+}$cells after seven-day culture was significantly higher in CML patients compared to the healthy controls $(3.07 \%$ vs. $0.21 \%$; $=0.001)$. The expression of CD14 measured as MFI (mean fluorescence intensity) was also higher on the cells generated from untreated CML patients $(\mathrm{p}=0.02)$.

The percentages of Mo-DC with expression of CD1a $(\mathrm{p}=0.002)$, CD80 ( $\mathrm{p}=0.0005), \mathrm{CD} 83$ $(0.0004), \mathrm{CD} 209(\mathrm{p}=0.02)$ as well as with co-expression of CD1a/CD83; $(\mathrm{p}=0.002)$, and CD80/ /CD86 (p = 0.04) were significantly lower in the CML patients group (Figure 1).

Phenotypic analysis also showed significantly lower expression of CD209 (MFI: 142.0 vs. 2790.0; $p=$ $=0.02)$ on $\mathrm{DC}$ from the CML patients compared to the healthy controls.

There was an adverse correlation between WBC count and the percentage of Mo-DC with co-expression of CD80 and CD86 $(R=-0.63 ; p=0.03)$.

In patients treated with TKI, in comparison with untreated CML patients, we observed higher efficacy of DC generation in seven-day cultures. The median percentage of DC among the cells in the culture was $30.7 \%$ (range: $3.22-82.89 \%$ ) and was significantly higher than in untreated patients.

The percentage of cells with CD14 expression was significantly lower in the patients treated with TKI $(p=0.0008)$, compared to untreated ones (Figure 1). Expression of CD209 on DC was higher compared to untreated patients with CML (MFI: 948.0 vs. 179.0; $\mathrm{p}=0.02$ ).
The time of TKI therapy correlated adversely with MFI for CD1a $(R=-0.49 ; p=0.006)$ and positively with MFI for CD83 $(\mathrm{R}=0.63 ; \mathrm{p}=0.01)$.

In nine patients, analysis performed before, and six months after, TKI therapy showed a significant decrease only in the percentage of $\mathrm{CD} 14^{+} \mathrm{Mo}-\mathrm{DC}$ $(\mathrm{p}=0.03)$. The expression of CD14 measured as MFI was also significantly lower $(p=0.03)$. In contrast, Mo-DC expressed a significantly higher amount of CD83 ( $p=0.03)$.

\section{Analysis of Treg cells in peripheral blood of CP-CML patients}

The percentage of $\mathrm{CD} 4{ }^{+} \mathrm{CD} 25^{\text {high }} \mathrm{FoxP}^{+}$cells was significantly higher in untreated CML patients compared to healthy controls $(\mathrm{p}=0.0002)$ (Figure 2). There was a significant correlation between the percentage of Tregs and the percentage of peripheral blood basophiles $(\mathrm{R}=0.821 ; \mathrm{p}<0.05)$. There was no significant change in the Treg percentage in CML patients after six months of TKI therapy.

\section{Analysis of Th17 cells percentages in peripheral blood of CP-CML patients}

The percentage of Th17 cells was significantly higher in untreated CML patients compared to healthy controls $(p=0.02)$ (Figure 3$)$. There was no significant change in the Th17 cells percentage in CML patients after six months of TKI therapy. But expression of intracellular IL-17 correlated negatively with the time of TKI therapy in the whole group of treated patients $(\mathrm{R}=-0.516 ; \mathrm{p}=0.02)$.

\section{Analysis of IL-6 level in serum of CP-CML patients}

There was no significant difference between the concentration of IL-6 in untreated CML patients and in the healthy controls. However, we noted a correlation between IL- 6 serum level and peripheral blood 

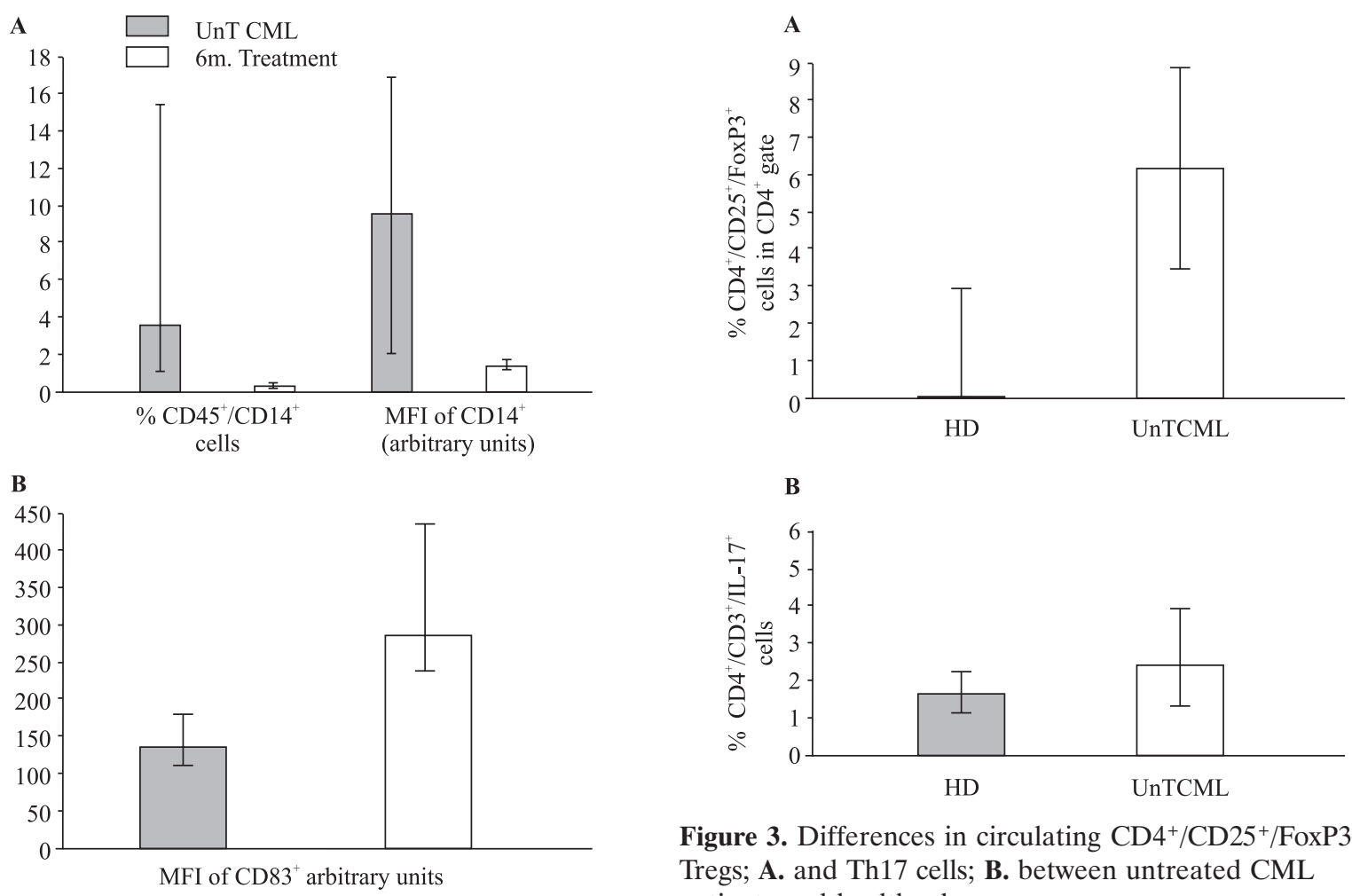

Figure 3. Differences in circulating $\mathrm{CD} 4^{+} / \mathrm{CD} 25^{+} / \mathrm{FoxP}^{+}$ Tregs; A. and Th17 cells; B. between untreated CML patients and healthy donors

Figure 2. Significant changes in the immunophenotype of Mo-DC after TKI treatment; A. percentage of CD $45^{+} \mathrm{CD} 14^{+}$cells and MFI of CD14; B. MFI of CD83; (UnT CML - before TKI treatment; $6 \mathrm{~m}$. Treatment after six months of TKI treatment)

WBC count $(\mathrm{R}=0.492 ; \mathrm{p}=0.04)$. There was also an inverse correlation between serum level of IL- 6 and the time of TKI therapy $(\mathrm{R}=-0.66 ; \mathrm{p}=0.03)$.

\section{Discussion}

Therapeutic approaches combining TKI with active DC immunotherapy might become a valuable option for patients with CML not achieving complete cytogenetic or molecular remission after TKI therapy. For the development of efficient immunotherapy protocols, detailed knowledge as to the effect of TKI on Mo-DC generation, as well as $\mathrm{T}$ cell populations, is required.

In this study, we compared the immunophenotype of Mo-DC generated from untreated patients with CML, from patients with CML treated with TKI, and from healthy donors.

We confirmed that DC could be efficiently differentiated from peripheral blood monocytes of CML patientxs $[9,14]$. The yield of generated DC was lower compared to healthy donors, with some differences noted in surficial markers, such as lower percentages of cells with expression of CD1a, CD80 and

CD83, CD206 and CD209. Previous studies on Mo-DC from CML patients have revealed divergent results. Eisendle et al. showed lower expression of CD80, CD83, CD86 on LPS maturated DC [4], which was improved when IL- $1 \beta$, TNF- $\alpha$ and PGE2 were used. DC cells maturated with a cytokine cocktail were fully functional in a mixed leukocyte reaction (MLR), something also found in a study by Westermann et al. [9]. Dong et al. showed high expression of maturation markers (CD80, CD86 HLA-DR) with IL-1 $\beta$ and TNF used for maturation. However, DC were defective in mannose receptor mediated endocytosis and macropinocytosis [15]. Significantly lower expression of CD206 (mannose receptor C type-1) and CD209 (DC-SIGN; DC-specific, ICAM-3 grabbing noninegrin) on Mo-DC maturated with TNF might be connected with impaired antigen capture observed by Dong et al.

These results suggest the need for further investigation to find the optimal generation conditions for Mo-DC from CML patients.

Results of Mo-DC generation from patients in complete cytogenetic remission after TKI therapy showed an improved yield of DC in seven-day cultures compared to the patients with active disease. We also found increased CD83 and CD209 expression, together with decreased CD1a expression. Most studies published so far have evaluated the effect of imatinib on DC generation when the drug was given 
to the culture of dendritic cells, but not in patients treated with imatinib. It has been shown that in the culture, imatinib impairs the differentiation of DC, both from $34^{+}$progenitor cells and monocytes, resulting in low expression of DC-associated cell surface molecules like CD1a and CD83, and costimulation molecules like CD80 and CD40 [16, 17]. Beside these phenotype alterations, the cells were unable to elicit primary T-cell responses as well as responses to recall antigens. Moreover, DC incubated with imatinib had a reduced endocytosis activity [16]. These in vitro observations have been confirmed by data from a mouse model [18]. In contrast, it has also been shown that imatinib does not affect CML-DC phenotype, migration profile or T-cell stimulatory capacity [19] and CML-DC display even improves antigen presentation abilities in the presence of imatinib [20]. In mice, DC treated with imatinib have exhibited enhanced APC function [21]. Limited studies investigating the impact of imatinib on naïve human blood DC have revealed that imatinib impairs neither the immunophenotype profile nor the DC-mediated polarization of naïve $\mathrm{CD}^{+} \mathrm{T}$ cells $[22,23]$.

In one study, where DC were generated from CML patients treated with TKI, there was an increase of CD1a, CD80, and CD86 compared to the parent population, but not to healthy donors. The authors showed that DC become BCR-ABL negative in CML patients treated successfully with imatinib [24], which might be advantageous because leukemic cells do not process antigen normally in CML [15]. It seems that more studies are needed into the immunophenotype and function of Mo-DC generated from patients treated with TKI in order to evaluate the use of Mo-DC immunotherapy in combination with TKI.

Next, we evaluated the percentage of T regulatory cells in CML patients, and found that it was significantly higher compared to healthy controls. Increased numbers of Tregs have previously been found in peripheral blood of cancer patients as well as in malignant effusions $[25,26]$. Recent studies have documented that Tregs suppress tumor-specific T-cell immunity, and therefore might contribute to the progression of human tumors [10]. There is very limited data regarding $\mathrm{T}$ regulatory cells in CML. Nadal et al. found that increased frequencies of Treg correlate with disease relapse after allogeneic stem cell transplantation [27]. However, increased populations of $\mathrm{CD} 4{ }^{+} \mathrm{CD} 25^{\text {high }}$ regulatory $\mathrm{T}$ cells have been found in acute myeloid leukemia (AML) patients $[28,29]$ and depletion of $\mathrm{T}$ regulatory cells dramatically improved the efficacy of DC vaccines in a preclinical AML murine model [30]. In the context of potential use of DC immunotherapy in patients treated with TKI, it would be useful to know how imatinib therapy affects Tregs. In our studies, there were no significant changes of Treg frequencies in imatinib-treated patients. However, in vitro studies showed that imatinib [31] and dasatinib impaired the proliferation and function of $\mathrm{CD} 4{ }^{+} \mathrm{CD} 25^{+}$regulatory cells in a dose-dependent manner [32]. Further studies into the function of Tregs in patients treated with TKI are needed, especially given that encouraging data in a mouse model showed that imatinib enhanced active immunotherapy against $\mathrm{BCR}-\mathrm{ABL}^{+}$tumors $[33,34]$.

Finally, we analyzed the percentages of Th17 cells in peripheral blood of CML patients. Th17 cells represent a novel subset of $\mathrm{T}$ helper cells that play an active role in inflammatory and autoimmune diseases [35]. Recent studies in solid tumors have also suggested the impact of Th17 cells, both in carcinogenesis and anti-cancer responses [36, 37]. Their role is probably complex and divergent, depending on the clinical stage and the role of angiogenesis and inflammation in tumor pathogenesis.

There has been no data published to date on Th17 in chronic myeloid leukemia. We found a significantly higher percentage of Th17 cells in untreated patients with CML than in healthy controls, and that this percentage decreased after six months of imatinib therapy. Though the decrease was not significant, there was a negative correlation between the expression of intracellular IL-17 in Th17 cells and the time of TKI therapy in the whole group of patients treated with TKI from four to 70 months. IL-6 belongs to important growth factors for Th17 in mice, and probably also in humans [38, 39]. Although we did not find a direct correlation between IL-6 serum level and Th17 cell percentage, there was a correlation between IL- 6 and WBC count in untreated CML patients, and a negative correlation between IL- 6 and the time of treatment in patients treated with TKI. Increased IL-6 level has been previously shown in CML patients $[40,41]$ and in patients with AML. Moreover, in AML patients, there was a positive correlation between high IL-6 concentration and increased percentage of Th17 cells $[42,43]$. High frequencies of Th17 cells were reduced when patients achieved complete remission after chemotherapy, suggesting that measurement of Th17 frequencies might have a clinical value in evaluating the effects of therapy. Such preliminary data suggests that Th17 cells might participate in the development of CML, although their role in this disease, and their potential role in immunotherapy, is yet to be established.

Taken together, our data shows that mature DC can be generated from CML patients treated with 
TKI, and that the yield of Mo-DC is higher compared to untreated patients, which should encourage further trials with DC immunotherapy in patients with cytogenetic response after TKI therapy. We also found increased frequencies of $\mathrm{T}$ regulatory and Th17 cells in CML patients, which might suggest a potential role in immunity from this disease. Further studies are needed to establish if manipulation of these populations might improve the results of DC immunotherapy.

\section{References}

1. Banchereau J, Steinman RM. Dendritic cells and the control of immunity. Nature. 1998;392:245-252.

2. Fazle Akbar SM, Abe M, Yoshida O, Murakami H, Onji M. Dendritic cell-based therapy as a multidisciplinary approach to cancer treatment: present limitations and future scopes. Curr Med Chem. 2006;13:3113-3119.

3. den Brok MH, Nierkens S, Figdor CG, Ruers TJ, Adema GJ. Dendritic cells: tools and targets for antitumor vaccination. Expert Rev Vaccines. 2005;4:699-710.

4. Eisendle K, Lang A, Eibl B et al. Phenotypic and functional deficiencies of leukaemic dendritic cells from patients with chronic myeloid leukaemia. Br J Haematol. 2003;120 63-73.

5. Deininger MW, Goldman JM, Melo JV et al. The molecular biology of chronic myeloid leukemia. Blood. 2000,96:3343-3356 .

6. Goldman JM. How I treat chronic myeloid leukemia in the imatinib era. Blood. 2007;110:2828-2837.

7. Fujii S, Shimizu K, Fujimoto K et al. Analysis of a chronic myelogenous leukemia patient vaccinated with leukemic dendritic cells following autologous peripheral blood stem cell transplantation. Jpn J Cancer Res. 1999;90:1117-1129.

8. Takahashi T, Tanaka Y, Nieda $\mathrm{M}$ et al. Dendritic cell vaccination for patients with chronic myelogenous leukemia. Leuk Res. 2003;27:795-802.

9. Westermann J, Kopp J, van Lessen A et al. Vaccination with autologous non-irradiated dendritic cells in patients with BCR/ $/ \mathrm{ABL}^{+}$chronic myeloid leukaemia. Br J Haematol. 2007;137: 297-306.

10. Beyer M, Schultze JL. Regulatory T cells: major players in the tumor microenvironment. Curr Pharm Des. 2009;15:1879_ -1892 .

11. Dannull J, Su Z, Rizzieri D, Yang BK et al. Enhancement of vaccine-mediated antitumor immunity in cancer patients after depletion of regulatory T cells. J Clin Invest. 2005;115:3623-3633 .

12. Harrington LE, Hatton RD, Mangan PR et al. Interleukin 17-producing $\mathrm{CD}^{+}+$effector $\mathrm{T}$ cells develop via a lineage distinct from the T helper type 1 and 2 lineages. Nat Immunol. 2005;6:1123-1132.

13. Martin-Orozco N, Dong C. The IL-17/IL-23 axis of inflammation in cancer: friend or foe? Curr Opin Investig Drugs. 2009;10:543-549.

14. Choudhury A, Gajewski JL, Liang JC et al. Use of leukemic dendritic cells for the generation of antileukemic cellular cytotoxicity against Philadelphia chromosome-positive chronic myelogenous leukemia. Blood. 1997;89:1133-1142.

15. Dong R, Cwynarski K, Entwistle A et al. Dendritic cells from CML patients have altered actin organization, reduced antigen processing, and impaired migration. Blood. 2003;101: 3560-3567.
16. Appel S, Boehmler AM, Grünebach F et al. Imatinib mesylate affects the development and function of dendritic cells generated from $\mathrm{CD} 34^{+}$peripheral blood progenitor cells. Blood. 2004;103:538-544.

17. Appel S, Rupf A, Weck MM et al. Effects of imatinib on monocyte-derived dendritic cells are mediated by inhibition of nuclear factor-kappaB and Akt signaling pathways. Clin Cancer Res. 2005;11:1928-1940.

18. Tad'eb J, Maruyama K, Borg C, Terme M, Zitvogel L. Imatinib mesylate impairs Flt3L-mediated dendritic cell expansion and antitumor effects in vivo. Blood. 2004;103:1966-1967.

19. Westers TM, Janssen JJ, Houtenbos I, Snoijs NC, van de Loosdrecht AA, Ossenkoppele GJ. Maintained immunogenicity of chronic myeloid leukemia-derived dendritic cells in the presence of imatinib mesylate: implication for vaccination regimens. Leukemia. 2006;20:154-157.

20. Sato N, Narita M, Takahashi M et al. The effects of STI571 on antigen presentation of dendritic cells generated from patients with chronic myelogenous leukemia. Hematol Oncol. 2003;21:67-75.

21. Wang H, Cheng F, Cuenca A et al. Imatinib mesylate (STI-571) enhances antigen-presenting cell function and overcomes tumor-induced CD4+ T-cell tolerance. Blood. 2005;105:1135-1143 .

22. Boissel N, Rousselot P, Raffoux E et al. Defective blood dendritic cells in chronic myeloid leukemia correlate with high plasmatic VEGF and are not normalized by imatinib mesylate. Leukemia. 2004;18:1656-1661.

23. Wehner R, Wendisch M, Schäkel K et al. Imatinib mesylate does not impair the immunogenicity of human myeloid blood dendritic cells. Leukemia. 2006;20:1629-1632.

24. Wang L, Butt NM, Atherton MG, Clark RE. Dendritic cells become BCR-ABL negative in chronic myeloid leukaemia patients successfully treated with imatinib. Leukemia. 2004;18:1025-1027.

25. Wolf AM, Wolf D, Steurer M, Gastl G, Gunsilius E, Grubeck-Loebenstein B. Increase of regulatory T cells in the peripheral blood of cancer patients. Clin Cancer Res. 2003;9:606-612.

26. Curiel TJ, Coukos G, Zou L et al. Specific recruitment of regulatory $\mathrm{T}$ cells in ovarian carcinoma fosters immune privilege and predicts reduced survival. Nat Med. 2004;10:942-949.

27. Nadal E, Garin M, Kaeda J, Apperley J, Lechler R, Dazzi F. Increased frequencies of CD4(+)CD25(high) $\mathrm{T}$ (regs) correlate with disease relapse after allogeneic stem cell transplantation for chronic myeloid leukemia. Leukemia. 2007;21:472-479.

28. Wang X, Zheng J, Liu J et al. Increased population of $\mathrm{CD} 4(+) \mathrm{CD} 25$ (high), regulatory T cells with their higher apoptotic and proliferating status in peripheral blood of acute myeloid leukemia patients. Eur J Haematol. 2005;75:46-476.

29. Szczepanski MJ, Szajnik M, Czystowska M et al. Increased frequency and suppression by regulatory $\mathrm{T}$ cells in patients with acute myelogenous leukemia. Clin Cancer Res. 2009;15: $3325-3332$.

30. Delluc S, Hachem P, Rusakiewicz S et al. Dramatic efficacy improvement of a DC-based vaccine against AML by CD25 $\mathrm{T}$ cell depletion allowing the induction of a long-lasting $\mathrm{T}$ cell response. Cancer Immunol Immunother. 2009;58:1669_ $-1677$.

31. Chen J, Schmitt A, Giannopoulos Ket al. Imatinib impairs the proliferation and function of $\mathrm{CD} 4{ }^{+} \mathrm{CD} 25^{+}$regulatory T cells in a dose-dependent manner. Int J Oncol. 2007;31:1133-1139.

32. Fei F, Yu Y, Schmitt A et al. Dasatinib inhibits the proliferation and function of $\mathrm{CD} 4+\mathrm{CD} 25+$ regulatory T cells. $\mathrm{Br}$ J Haematol. 2009;144:195-205. 
33. Zeng Y, Graner MW, Feng H, Li G, Katsanis E et al. Imatinib mesylate effectively combines with chaperone-rich cell lysateloaded dendritic cells to treat $\mathrm{BCR}-\mathrm{ABL}^{+}$murine leukemia. Int J Cancer. 2004;110:251-259.

34. Larmonier N, Janikashvili N, LaCasse CJ et al. Imatinib mesylate inhibits $\mathrm{CD} 4{ }^{+} \mathrm{CD} 25^{+}$regulatory $\mathrm{T}$ cell activity and enhances active immunotherapy against BCR-ABL tumors. J Immunol. 2008;181:6955-6963.

35. Romagnani S. Human Th17 cells. Arthritis Res Ther. 2008; 10:206.

36. Wang L, Yi T, Kortylewski M et al. IL-17 can promote tumor growth through an IL-6-Stat3 signaling pathway. J Exp Med. 2009;206:1457-1464.

37. Benchetrit F, Ciree A, Vives V. Interleukin-17 inhibits tumor cell growth by means of a T-cell-dependent mechanism. Blood. 2002;99:2114-2121.

38. Bettelli E, Korn T, Oukka M, Kuchroo VK et al. Induction and effector functions of T(H)17 cells. Nature. 2008;453:1051-1057.
39. Volpe E, Servant N, Zollinger R et al. A critical function for transforming growth factor-beta, interleukin 23 and proinflammatory cytokines in driving and modulating human $\mathrm{T}(\mathrm{H})-17$ responses. Nat Immunol. 2008;9:650-657.

40. Humlová Z, Klamová H, Janatková I et al. Immunological profiles of patients with chronic myeloid leukaemia. I. State before the start of treatment. Folia Biol (Praha). 2006;52:47-58.

41. Panteli KE, Hatzimichael EC, Bouranta PK et al. Serum interleukin (IL)-1, IL-2, sIL-2Ra, IL-6 and thrombopoietin levels in patients with chronic myeloproliferative diseases. $\mathrm{BrJHae}$ matol. 2005;130:709-715.

42. Thomas X, Hirschauer C, Troncy J et al. Serum interleukin-6 levels in adult acute myelogenous leukemia: relationship with disease characteristics and outcome. Leuk Lymphoma. 1997;24: 291-300.

43. Wu C, Wang S, Wang $\mathrm{F}$ et al. Increased frequencies of $\mathrm{T}$ helper type 17 cells in the peripheral blood of patients with acute myeloid leukaemia. Clin Exp Immunol. 2009;158:199-204.

Submitted: 20 June, 2010 Accepted after reviews: 21 November, 2010 